EPJ Web of Conferences 59, 02019 (2013)

DOI: $10.1051 /$ epjconf/20135902019

(C) Owned by the British Crown, published by EDP Sciences, 2013

\title{
Sensitivity of ICF ignition conditions to non-Maxwellian DT fusion reactivity
}

\author{
W.J. Garbett ${ }^{\mathrm{a}}$
}

AWE plc, Aldermaston, Reading, Berkshire RG7 4PR, UK

\begin{abstract}
The hotspot ignition conditions in ICF are determined by considering the power balance between fusion energy deposition and energy loss terms. Uncertainty in any of these terms has potential to modify the ignition conditions, changing the optimum ignition capsule design. This paper considers the impact of changes to the DT fusion reaction rate due to non-thermal ion energy distributions. The DT fusion reactivity has been evaluated for a class of non-Maxwellian distributions representing a perturbation to the tail of a thermal distribution. The resulting reactivity has been used to determine hotspot ignition conditions as a function of the characteristic parameter of the modified distribution.
\end{abstract}

\section{INTRODUCTION}

The hotspot ignition conditions in ICF can be determined by considering the power balance between fusion alpha particle energy deposition, hydrodynamic work and energy loss via radiation and electron thermal conduction [1]. The resulting conditions may be sensitive to uncertainties in any of the key plasma physics models or assumptions. This paper considers the impact of changes to the DT fusion reaction rate due to the presence of non-thermal ion energy distributions.

The reactivity, $\langle\sigma v\rangle$, for thermonuclear fusion reactions is conventionally derived by averaging crosssection data over a Maxwell-Boltzmann energy distribution. In many situations, such as when plasmas are strongly shocked or rapidly heated by energetic particles, reacting ions may be better characterised by a non-Maxwellian distribution. Since the self-relaxation time is longer for ions at higher energies [2], such effects typically produce an enhancement or depletion within the tail of the distribution. At temperatures where the reactivity is dominated by the contribution of particles in the tail, even small changes to the distribution may have a potentially significant impact on the fusion reaction rate.

The DT fusion reactivity has been evaluated for a class of non-Maxwellian distributions, the q-Gaussian distribution [3], which represent a perturbation to the tail of a thermal distribution. The resulting reactivity has been used to calculate a revised ideal ignition temperature [4] and to determine hotspot ignition conditions as a function of the characteristic parameter of the distribution. This initial assessment does not consider in detail the physical development of non-Maxwellian distributions. If conditions are found to show significant sensitivity, then the generation of distributions may be explored in future work via kinetic simulation.

\section{Q-GAUSSIAN DISTRIBUTION}

The q-Gaussian distribution [3] provides a convenient single-parameter representation of deviations from a Maxwellian distribution:

$$
f_{q}\left(E, T^{\prime}\right) \sim\left(\frac{1}{k T^{\prime}}\right)^{3 / 2} E^{1 / 2}\left[1-(1-q) \frac{E}{k T^{\prime}}\right]^{1 /(1-q)} .
$$

\footnotetext{
ae-mail: warren.j.garbett@awe.co.uk
}

This is an Open Access article distributed under the terms of the Creative Commons Attribution License 2.0, which permits unrestricted use, distribution, and reproduction in any medium, provided the original work is properly cited. 

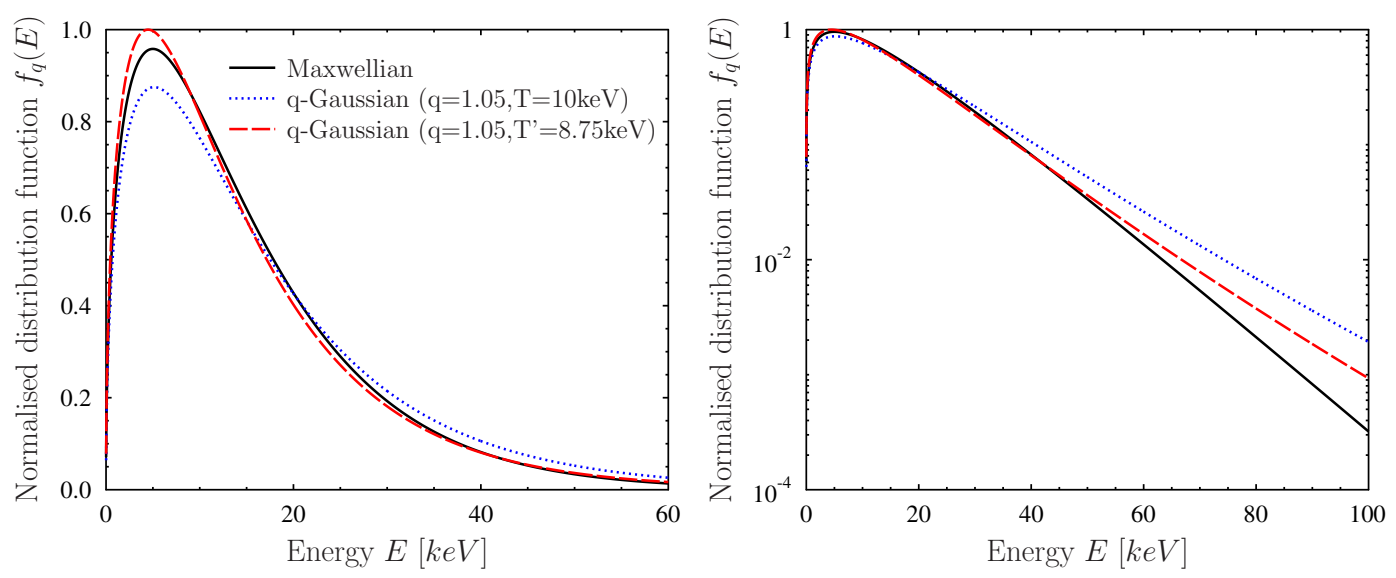

Figure 1. Maxwellian ion distribution at $T=10 \mathrm{keV}$ (solid) and q-Gaussian distributions with $q=1.05$, at the same temperature (dotted) and at the effective temperature $T^{\prime}=8.75 \mathrm{keV}$ given by equation 2 (dashed). Distributions are normalised to the peak amplitude of the dashed curve.

A Maxwellian is recovered as $q \rightarrow 1$. It is important to conserve the total energy of the distribution to ensure fair comparison with an equivalent Maxwellian. This is achieved by defining the equivalent temperature $T^{\prime}$ such that:

$$
\left\langle E\left(T^{\prime}\right)\right\rangle_{q}=\langle E(T)\rangle_{M B}=\frac{3}{2} k T .
$$

A comparison of Maxwellian and q-Gaussian distributions is shown in Figure 1, which illustrates the increase in tail population for $q>1$. Note that a q-Gaussian evaluated at the Maxwellian temperature $T$ will overestimate the enhancement to the tail. Energy conservation also gives rise to a small shift in the energy of the peak.

To provide a physical interpretation of the $q$ parameter it is useful to relate $q$ to the change in population of the distribution tail. A natural and consistent definition of the tail arises by considering the ion energy $E_{0}$ at which Maxwellian and q-distribution amplitudes are equal. It is found that $\left(\frac{E_{0}}{k T}\right) \approx 4$, independent of $T$ and almost independent of $q$. The relation between $q$ and the change in tail population derived using this definition is shown in Figure 2.

\section{EVALUATION OF DT REACTIVITY}

The DT fusion reactivity has been evaluated for a q-Gaussian distribution over a range of $q$ values (Figure 2). Significant deviation from Maxwellian reactivity is observed at lower temperatures $(\sim 1 \mathrm{keV})$, where the reactivity is dominated by a very small fraction of the ion population, in the tail of the distribution. As the temperature increases through $10 \mathrm{keV}$ the deviation is reduced, as the reactivity becomes less weighted by the tail of the distribution. At still higher temperatures $(\sim 30 \mathrm{keV})$ the qGaussian correction remains small, but is reversed relative to that at lower temperatures. At these high temperatures the peak reactivity now corresponds to the body of the ion distribution. The body of a $q>1$ (enhanced tail) q-Gaussian is actually depleted relative to a Maxwellian as a consequence of particle and energy conservation.

Convergence of the reactivity curves at higher temperature suggests that ignition capsules will show little sensitivity to the ion distribution once they are lit. However, prior to the onset of ignition, during the $5-10 \mathrm{keV}$ self-heating phase, the impact could potentially be significant. At $5 \mathrm{keV}$ a $15 \%$ change 

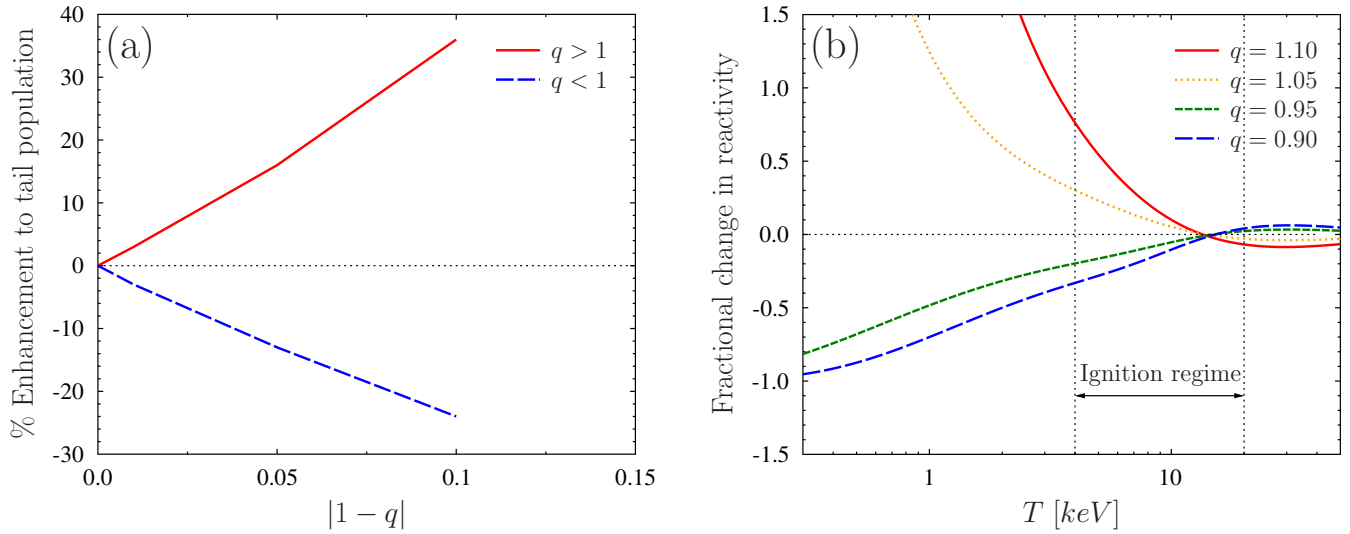

Figure 2. (a) Relation between $q$ parameter and change in tail population. (b) Fractional change in DT reactivity for different $q$ values compared to nominal Maxwellian case.
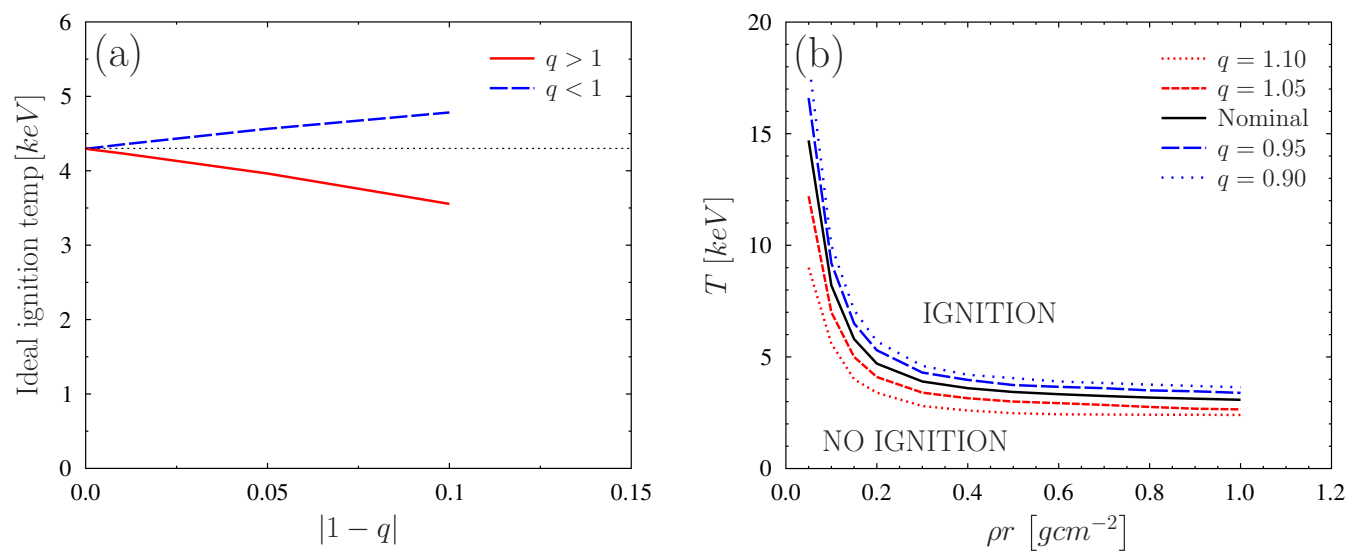

Figure 3. (a) Ideal ignition temperature evaluated for different values of the $q$ parameter. (b) Boundary of hotspot ignition conditions derived for different $q$ values using 1D simulations of a simple isobaric fuel configuration.

in the tail population gives $\sim 20 \%$ change in reactivity. It is also possible that non-Maxwellian reactivity effects could be observable in cooler non-ignition implosion experiments.

\section{IGNITION CONDITIONS}

The ideal ignition temperature is defined by the balance of alpha deposition and bremsstrahlung radiation loss [4]:

$$
W_{b} \equiv A_{b} n_{e}^{2} T^{1 / 2}=W_{\alpha} \equiv \frac{1}{4} n_{i}^{2}\langle\sigma v\rangle Q_{\alpha}
$$

Assuming fully ionised, optically thin plasma gives a density-independent ignition temperature. Figure 3 shows the ideal ignition temperature evaluated for different $q$. The ignition temperature is less sensitive to $q$ than the fusion reactivity. This is a result of the strong temperature dependence of the fusion reactivity; a significant change in alpha power deposition can be negated by only a small change in temperature. Mathematically, approximating $\langle\sigma v\rangle$ locally as $\sim a T^{n}$, with $n \sim 3.8$ over the range 
$3.5-4.8 \mathrm{keV}$ gives:

$$
T_{q}^{i g} \sim T_{0}^{i g}\left(\frac{a_{q}}{a_{0}}\right)^{-0.3}
$$

Hence at $q=0.95$ a reduction of $18 \%$ in $\langle\sigma v\rangle$ gives an increase of only $6 \%$ in ignition temperature.

Hotspot ignition conditions for an isobaric fuel configuration were evaluated for different $q$ using Nym radiation-hydrocode simulations in 1D [5]. For each $q$ value a modified DT reactivity versus temperature table was generated off-line and inserted into the code. The calculations allowed for separate electron, ion and radiation temperatures and included alpha particle transport and radiation diffusion. The boundary of hotspot ignition conditions is shown for different $q$ in Figure 3. The fractional change in ignition temperature was found to be approximately independent of $\rho r$, and is a function of $q$ only. For a NIF-like $\rho r \sim 0.5 \mathrm{gcm}^{-2}$ the ignition temperature was found to vary by only $\sim 0.5 \mathrm{keV}$, even allowing for considerable changes in the tail population.

\section{CONCLUSIONS}

The impact of non-thermal ion energy distributions on the DT fusion reaction rate and ICF ignition conditions has been considered. The DT reactivity evaluated using the q-Gaussian distribution can show significant sensitivity at $T<10 \mathrm{keV}$, particularly at lower temperatures $(\sim 1 \mathrm{keV})$ where the reactivity is dominated by the contribution of particles in the tail of the distribution. However, the ideal ignition temperature and hotspot ignition conditions are considerably less sensitive than the DT reactivity. Although only one class of distributions has been considered, the results suggest that the impact of non-Maxwellian distributions is unlikely to be a first order effect for NIF-scale ignition targets.

\section{References}

[1] J.D. Lindl, Phys. Plasmas 2, 3933 (1995)

[2] W.M. MacDonald, M.N. Rosenbluth, W. Chuck, Phys. Rev. 107, 350 (1957)

[3] C. Tsallis, J. Stat. Phys. 52, 479 (1988)

[4] S. Atzeni, J. Meyer-ter-vehn, The Physics of Inertial Fusion, Oxford (2004)

[5] P.D. Roberts, S.J. Rose, P.C. Thompson, R.J. Wright, J. Phys. D 13, 1957 (1980) 